\title{
Study on the interaction process of ozone-treated Direct Red 80 and Human serum albumin at the molecular level
}

\author{
Yan Song ${ }^{1}$, Yuzhi Shi ${ }^{21}$, Kejia Zhang ${ }^{2}$ \\ ${ }^{1}$ School of Water Conservancy and Environment, University of Jinan, Jinan, Shandong Province,250022 \\ ${ }^{2}$ Water Resources Research Institute of Shandong Province, Jinan,250014, China
}

\begin{abstract}
In recent years, as the production and use of dyes have been greatly developed, the treatment of dye wastewater has become one of the current environmental problems that must be resolved. Ozone (O3) is a strong oxidant and is widely used in water treatment. In this study, the interaction process of ozone-treated Direct Red 80 (otDR80) and human serum albumin (HSA) was explored using multi-spectra methods. The results showed that otDR80 made the skeleton structure of HSA loosened and unfolded. OtDR80 changed the secondary structure of HSA, making the chromophore in the hydrophobic interior of HSA more tightly packed. The intensity of RLS spectra showed a upward trend after exposure to otDR80, indicating that the size of the reaction system increased. This study provides a new method for the degradation of DR80 and its toxicological study at the molecular level.
\end{abstract}

\section{Introduction}

The printing and dyeing industry is a typical industry with high energy and water consumption, and it is also one of the key polluting industries in my country. In recent years, with the development of the dye industry, dyes have been more widely used in industry and textile industries, and a large amount of dye wastewater has been produced. Although the use of this dye has been banned in some countries and has not been approved by the US Food and Drug Administration, it is still used in many parts of the world.

$\mathrm{O}_{3}$ is a strong oxidant, its oxidizing ability is second only to fluorine, and 1.52 times that of elemental chlorine, and it is widely used in water treatment. It can react with aromatic azo compounds to break the azo bond, thereby degrading it. There are two main oxidation paths of $\mathrm{O}_{3}$, namely direct oxidation of ozone molecules and indirect oxidation mainly based on hydroxyl radicals. Because ozone is a highly effective decolorant and oxidant, many factories will use ozone to pre-treat DR80 dye wastewater before discharging to meet emission standards. The current studies focus on the degradation and decolorization effects of DR80[1,2]. However, there are few studies on the interaction mechanism of DR80 and proteins and the effect of ozone treatment on this process. In this studies, Human serum albumin (HSA) was selected as the protein model because of its low cost, ready availability, and unusual ligand-binding properties.

HSA plays an important role in maintaining plasma osmotic pressure, blood volume, $\mathrm{pH}$ and osmotic pressure[3]. It consists of three domains of I (1-195 amino acids), II (196-383 amino acids), III (384-585 amino acids) which have similar $\alpha$-helical structure[4], containing 585 amino acids, each domain is connected by a disulfide bond, and each domain is composed of two structural subdomains, and the spatial structure is heart-shaped[5]. Albumin is unique in that it binds to a variety of compounds such as metabolites, drugs, dyes and fatty acids[6].

In this paper, the interactions process of ozone-treated DR80 (otDR80) and HSA were explored using multispectra methods and the research flow chart was shown in Fig.1(a).

\section{Materials and methods}

\subsection{Chemicals and regents}

The DR80 was got from Xingtai Caiyuan Chemical Co., LTD, with a molecular weight of 1373 and a molecular formula of $\mathrm{C} 45 \mathrm{H} 26 \mathrm{~N} 10 \mathrm{Na} 6 \mathrm{O} 21 \mathrm{~S} 6$. When used, it was formulated into a corresponding concentration of stock solution as needed. The buffer solution was selected to be $0.2 \mathrm{M} \mathrm{NaH} 2 \mathrm{PO} 4 / \mathrm{Na} 2 \mathrm{HPO} 4$ solution with $\mathrm{pH}=7.4$ (0.2M $\mathrm{NaH} 2 \mathrm{PO} 4 \cdot 2 \mathrm{H} 2 \mathrm{O}$ and $\mathrm{Na} 2 \mathrm{HPO} 4 \cdot 12 \mathrm{H} 2 \mathrm{O})$.

\footnotetext{
*The corresponding author: Yuzhi Shi: 1289143995@qq.com
} 
(a)

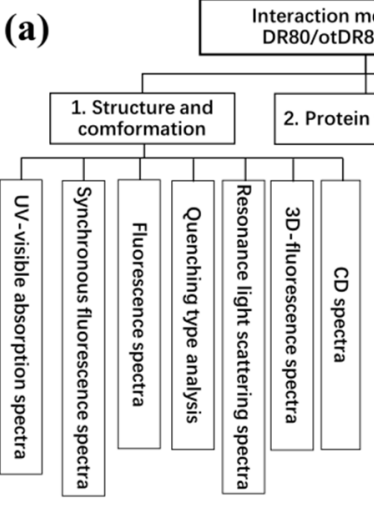

(b)

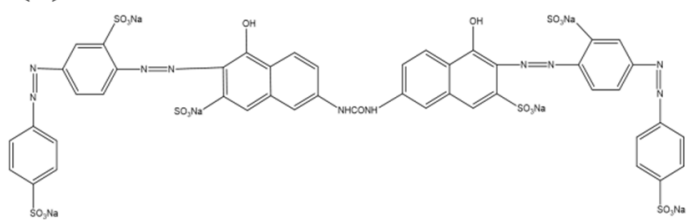

Fig.1 Interaction mechanisms of DR80 with HSA (a) and the molecular structure of DR80(b).

\subsection{Apparatus and methods}

The experiment used $1 \mathrm{~mL}$ phosphate buffer, $1 \mathrm{~mL}$ HSA solution and $1 \mathrm{~mL}$ of different concentration and different oxidation degree of DR80 diluted with ultrapure water in a $10 \mathrm{~mL}$ colorimetric tube, and then reacted at the required temperature after 40 minutes to ensure the system was equilibrated.

\subsection{Fluorescence analysis}

Three-dimensional fluorescence spectra of the experimental system were scanned using an F-4600 spectrofluorometer and a $1 \mathrm{~cm}$ optical diameter cuvette. The excitation wavelength was set at $200-400 \mathrm{~nm}$, the emission wavelength was set at $200-500 \mathrm{~nm}$, the excitation and emission slit spectral width was $5 \mathrm{~nm}$, the excitation and emission wavelength steps were $5 \mathrm{~nm}$, the scanning speed was set at $12,000 \mathrm{~nm} / \mathrm{min}$, and the photomultiplier detector voltage was $700 \mathrm{~V}$.

For fluorescence spectroscopy and synchronous fluorescence spectroscopy, the inner filter effect (IFE) may cause the deviation of the fluorescence intensity, so the IFE needs to be corrected when analyzing the fluorescence results[7,8]. The following equation was used to correct the fluorescence intensity in this study.

$$
\mathrm{F}_{\text {cor }}=\mathrm{F}_{\mathrm{obs}} \times 10^{\left(\mathrm{A}_{1}+\mathrm{A}_{2}\right)} / 2
$$

where Fcor and Fobs are the fluorescence intensity before and after the correction; $\mathrm{A} 1$ and $\mathrm{A} 2$ are the absorption values of the system at the excitation and relative emission wavelengths.

\subsection{UV-vis analysis}

The UV-Vis absorption spectra of the system were measured using a UV-Vis spectrophotometer
(SHIMADZU UV-2600). Use a cuvette with optical diameter of $1 \mathrm{~cm}$, wavelength range of $190-900 \mathrm{~nm}$, slit width set as $2 \mathrm{~nm}$, and use ultrapure water for zero setting.

\subsection{Circular dichroism spectra}

The circular dichroism spectrum of the experimental system was scanned with a $1 \mathrm{~mm}$ path length quartz cell, purged with nitrogen, the instrument response time was 1 $\mathrm{s}$, the scanning range was set at $190-260 \mathrm{~nm}$, the scanning speed was set at $200 \mathrm{~nm} / \mathrm{min}$, the data acquisition interval was selected as $0.5 \mathrm{~nm}$, each spectrum was scanned three times, and its average value was taken. The signal value of $\mathrm{NaH} 2 \mathrm{PO} 4 / \mathrm{Na} 2 \mathrm{HPO} 4$ buffer was scanned for background subtraction at the time of measurement.

\section{Result and discussions}

\subsection{Effect of otDR80 on the skeleton structure of HSA}
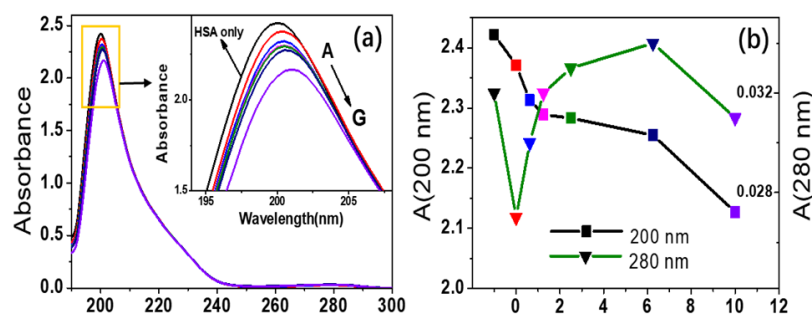

Fig.2 Effect of otDR80 on the UV-vis spectra of HSA.

(a) UV-vis spectra; (b) The scatter plots of UV-vis spectra under different ozone dosage;

Conditions: $\mathrm{T}=310 \mathrm{~K}, \mathrm{pH}=7.4,[\mathrm{HSA}]=1 \mu \mathrm{M},[\mathrm{DR} 80]=10 \mu \mathrm{M}$

Ozone dosage to DR80: $(\mathrm{A} \rightarrow \mathrm{G})=\mathrm{HSA}$ only, $0,0.62,1.25,2.5,6.25,10 \mathrm{~g} / \mathrm{L}$.

In order to investigate and compare the effect of otDR80 on the structure and conformation of HSA, we measured the changes of UV-vis absorption spectra of HSA with DR80 which was treated in different dosage of ozone. As can be seen in Fig.2(a/b), the intensity of the absorption peak at $210 \mathrm{~nm}$ showed a downward trend with an obvious red shift, which was same as the dosedependent result. This phenomenon demonstrated that otDR80 made the skeleton structure of HSA loosened and unfolded and we speculated that the changes in the UV-Vis absorption spectra was caused by the composite effect of the ozone treatment products of DR80[9]. Nevertheless, Fig.2(b) showed that the intensity of absorption peak around $280 \mathrm{~nm}$ had no regular change, hence we used the fluorescence spectra to detect the changes of HSA amino acid residue in the following section.

\subsection{Effect of otDR80 on the secondary structure contents of HAS}

Table.1 Effects of otDR80 on HSA secondary structure

Ozone Secondary structure content in HSA (\%) 


\begin{tabular}{|c|c|c|c|c|}
\hline $\begin{array}{c}\text { dosage } \\
(\mathbf{g} / \mathbf{L})\end{array}$ & $\boldsymbol{\alpha}$-Helix & $\boldsymbol{\beta}$-Sheet & $\boldsymbol{\beta}$-Turn & Unordered \\
\hline 0 & 43.5 & 6.7 & 19.1 & 32.6 \\
\hline 0.94 & 45.2 & 8.1 & 18.3 & 31.9 \\
\hline 10 & 49.9 & 5.0 & 14.5 & 31.8 \\
\hline
\end{tabular}

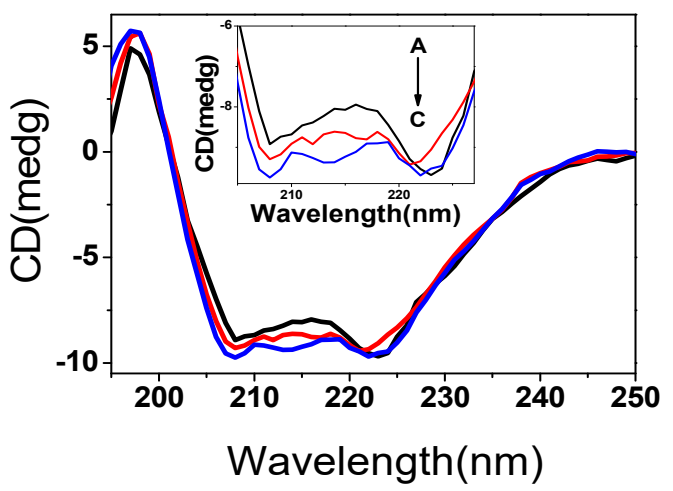

Fig.3 Effect of otDR80 on the CD spectra of HSA. Conditions: $\mathrm{T}=310 \mathrm{~K}, \mathrm{pH}=7.4$, [HSA] $=1 \mu \mathrm{M}$, [DR80] $=10$ $\mu \mathrm{M}$; Ozone dosage to DR80: $(\mathrm{A} \rightarrow \mathrm{C})=0,0.94,10 \mathrm{~g} / \mathrm{L}$;

The CD spectra of HSA under exposure of otDR80 which shown in Fig.3 and Table.1 displayed the contents change of HSA secondary structure. It was clearly that two negative bands at $209 \mathrm{~nm}$ and $218 \mathrm{~nm}$ showed a downward trend after treatment of the otDR 80 , we can see that the content of HSA $\alpha$-helical structure increased by $6.4 \%$ while the contents of $\beta$-sheet, $\beta$-turn and unordered curling decreased by $1.7 \%, 4.6 \%$ and $0.8 \%$ respectively. This result indicated that after ozone treatment, DR80 and its ozone oxidized products changed the secondary structure of HSA, making the chromophore in the hydrophobic interior of HSA more tightly packed. In addition, The CD spectra peaks of HSA exposed to otDR80 only showed a downward trend in intensity, no shift of the peaks position, and no new peaks were generated during the interaction, indicating that DR80 and its ozonization products only changed the secondary structure content without disrupting the overall conformation of the protein.

\subsection{Effect of otDR80 on the protein size of HSA}

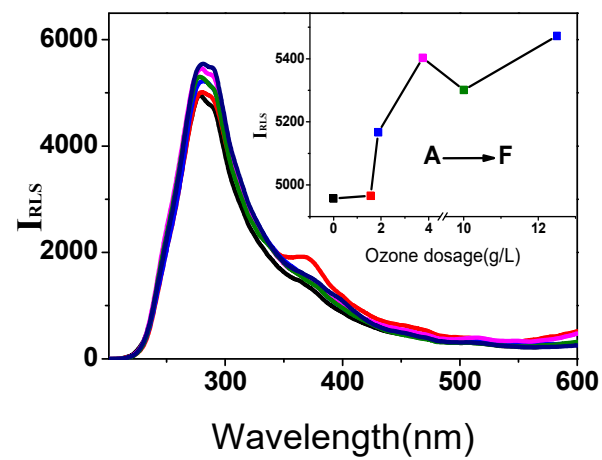

Fig.4 Effect of otDR80 on the RLS spectra of HSA. Conditions: $\mathrm{T}=310 \mathrm{~K}, \mathrm{pH}=7.4$, [HSA $]=1 \mu \mathrm{M},[\mathrm{DR} 80]=10$ $\mu \mathrm{M}$; Ozone dosage to DR80:(A $\rightarrow \mathrm{F})=0,1.56,1.88,3.75,10,12.5$ $\mathrm{g} / \mathrm{L}$

Fig.4 showed the RLS spectra of HSA under the exposure of otDR 80 and the inset was the scatter plot of
RLS peak intensities under different ozone dosage. The intensity of RLS spectra showed a upward trend after exposure to otDR80, indicating that the size of the reaction system increased. It was worth noting that the intensity of the system RLS is positively correlated with the dosage of ozone added, which might be because the more thorough the oxidation, the more small molecule intermediates were produced, and these small molecule intermediates were more likely to form aggregates with $\operatorname{HSA}[10,11]$.

\subsection{Investigations of HSA amino acid residue changes after otDR80 treatment}
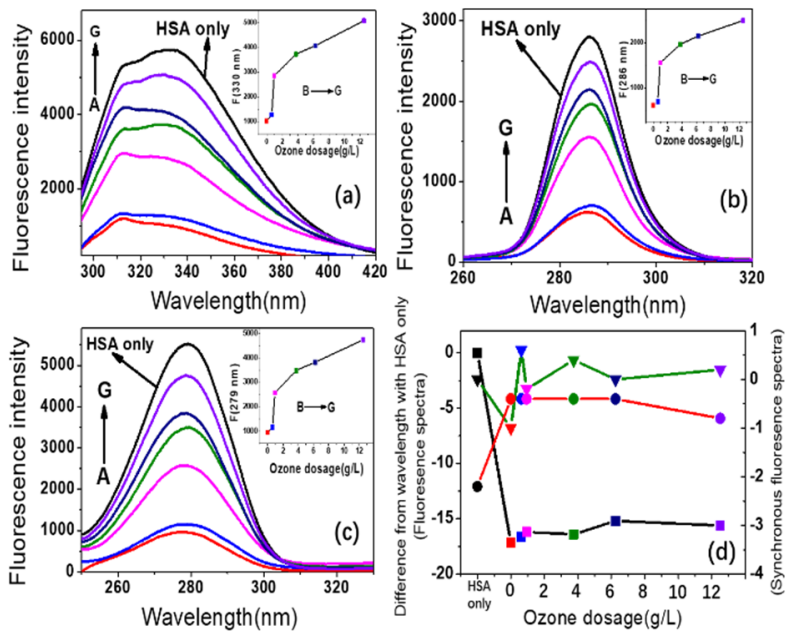

Fig.5 Effect of otDR80 on amino acid residue of HSA.

(a) Fluorescence spectra of HSA. (b) Synchronous fluorescence $\operatorname{spectra}(\Delta \lambda=15 \mathrm{~nm})$ of HSA. (c) Synchronous fluorescence spectra $(\Delta \lambda=60 \mathrm{~nm})$ of HSA. (d) Difference in wavelength of fluorescence and synchronous fluorescence spectra from the sample of HSA only.

Conditions: $\mathrm{T}=310 \mathrm{~K}, \mathrm{pH}=7.4$, [HSA] $=1 \mu \mathrm{M}$; Ozone dosage to $\mathrm{DR} 80(\mathrm{~A} \rightarrow \mathrm{G})=0,0.62,0.94,3.75,6.25,12.5$, HSA only $(\mathrm{g} / \mathrm{L})$.

The effect of otDR80 on HSA fluorescence spectra is shown in Fig.5(a). The results showed that with the progress of ozonization, the fluorescence quenching degree of HSA caused by Direct Red 80 was gradually weakened, and the peak values were rising. During the ozone oxidation, the composite effect of ozonization products of DR80 made the chromophore in the hydrophobic interior of HSA more tightly packed[12]. This is similar to micellar sensitization, which increased the fluorescence quantum yield, reduced the energy loss and the degree of fluorescence quenching of collision between small molecules and chromophores, thus increasing the fluorescence intensity of the entire system[5].

The synchronous fluorescence spectra of the otDR80HSA system showed the same tendency as the fluorescence spectra (Fig. 5 b/c). It can be seen that the otDR80 increased the fluorescence intensity of tyrosine(Tyr), but the highest peak did not shift regularly, indicating that the otDR80 could not affect the microenvironment where tyrosine(Tyr) was located. For tryptophan(Trp), it is characterized by fluorescence enhancement with a slight red shift (see Fig.5d), indicating that the microenvironment of the tryptophan(Trp) residue 
was enhanced in hydrophobicity and its polarity was weakened[13].

\subsection{Effect of DR80 and otDR80 on Three- dimensional fluorescence spectra of HSA.}

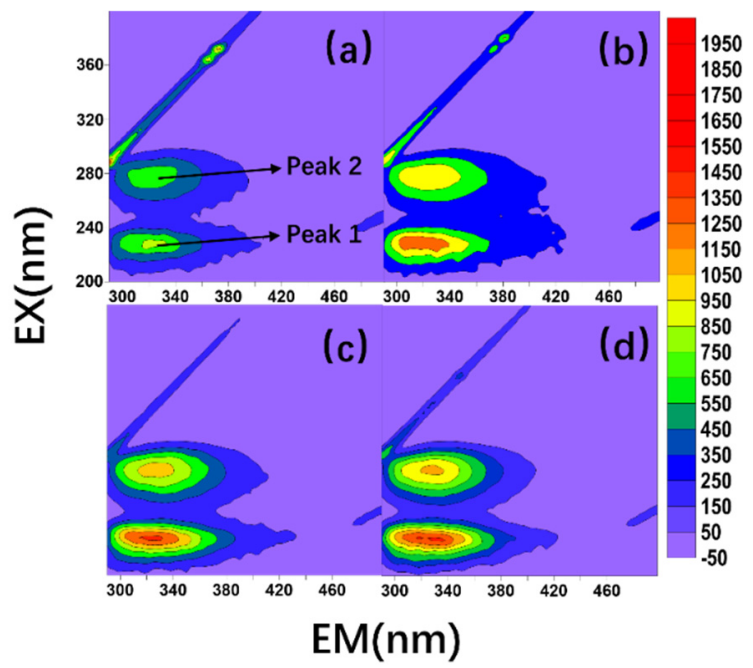

Fig.6 Effect of otDR80 on three-dimensional fluorescence spectra of HSA.

Conditions: $\mathrm{T}=310 \mathrm{~K}, \mathrm{pH}=7.4$, [HSA] $=1 \mu \mathrm{M}$ (a-d) Ozone dosage to DR80 $=0.21,0.62,6.25,12.5 \mathrm{~g} / \mathrm{L}$; [DR80] $=10 \mu \mathrm{M}$

Table.2 Three-dimensional fluorescence information of otDR80 combined with HSA

\begin{tabular}{|c|c|c|c|c|}
\hline & Peak1 & \multicolumn{2}{|c|}{ Peak2 } \\
\hline $\begin{array}{c}\text { Ozone } \\
\text { dosage } \\
(\mathbf{g} / \mathbf{L})\end{array}$ & $\begin{array}{c}\text { Position } \\
\lambda \mathbf{e x} / \boldsymbol{\lambda} \text { em } \\
(\mathbf{n m} / \mathbf{n m})\end{array}$ & $\begin{array}{c}\text { Intensity } \\
\mathbf{F}\end{array}$ & $\begin{array}{c}\text { Position } \\
\boldsymbol{\lambda} \mathbf{e x} / \boldsymbol{\lambda} \mathbf{e m} \\
(\mathbf{n m} / \mathbf{n m})\end{array}$ & $\begin{array}{c}\text { Intensity } \\
\mathbf{F}\end{array}$ \\
\hline 0.21 & $228 / 326$ & 445.33 & $276 / 322$ & 368.37 \\
0.62 & $228 / 326$ & 681.83 & $276 / 318$ & 504.29 \\
6.25 & $228 / 330$ & 1157.43 & $280 / 334$ & 830.74 \\
12.5 & $224 / 338$ & 199.77 & $276 / 334$ & 1120 \\
\hline
\end{tabular}

Three-dimensional fluorescence spectroscopy is an emerging means to analyze protein conformational changes[14]. In order to investigate the effect of DR80 and otDR80 on HSA structure and conformation, the threedimensional fluorescence spectra of the two system were scanned. The results was shown in Fig. 6 and Table.2.

As can be seen from Fig.6(a-d) and Table. 2, peak 1 $(\lambda \mathrm{ex}=225-235 \mathrm{~nm} / \lambda \mathrm{em}=305-340 \mathrm{~nm})$ can reflect the characteristic absorption peak of the HSA polypeptide chain skeleton structure of $\mathrm{C}=\mathrm{O}$ while peak $2(\lambda \mathrm{ex}=275$ $285 \mathrm{~nm} / \lambda \mathrm{em}=310-340 \mathrm{~nm})$ represents the information of the tryptophan(Trp) microenvironment. The peak of $\lambda \mathrm{em}$ $=\lambda$ ex represents the first Raleigh scattering peak[15]. As the oxidation of ozone progresses, both peak 1 and peak 2 showed a upward trend in intensity, and peak 2 was accompanied by a blue shift in the emission wavelength, indicating that after oxidation, the composite effect of ozonization products changed the HSA skeleton structure, and the microenvironment of the tryptophan(Trp) residue is enhanced in hydrophobicity and its polarity is weakened, which is consistent with the results of fluorescence results.

\section{Conclusions}

In this paper, the interaction process of ozone-treated Direct Red 80 and human serum albumin was explored using mult-spectra methods. The otDR80 made the skeleton structure of HSA loosened and unfolded. The small molecule intermediates of DR80 after ozonization were more likely to form aggregates with HSA. The otDR80 made the fluorescent chromophores in the interior of HSA more tightly packed. In addition, after oxidation, the composite effect of ozonization products changed the microenvironment of the tryptophan(Trp) residue, enhanced its hydrophobicity. This study provides a new method for the degradation of DR80 and its toxicological study at the molecular level.

\section{Acknowledgements}

This study was supported by the National Key Research and Development Program of China (NO. 2016YFC0402809, 2018YFC0408000, 2018YFC0408006); the Shandong Water Scientific Research and Technology Extension Project (Grant No. SDSLKY201703) and the Technology Demonstration Project of Ministry of Water Resources of China (NO. SF201803).

\section{Reference:}

1. Mahmoodi, N.M.; Arami, M.; Limaee, N.Y.; Tabrizi, N.S. Decolorization and aromatic ring degradation kinetics of Direct Red 80 by UV oxidation in the presence of hydrogen peroxide utilizing $\mathrm{TiO} 2$ as a photocatalyst. Chemical Engineering Journal 2005, 112, 191-196.

2. Lopes, A.; Martins, S.; Morao, A.; Magrinho, M.; Gonçalves, I. Degradation of a textile dye CI Direct Red 80 by electrochemical processes. Portugaliae Electrochimica Acta 2004, 22, 279-294.

3. Maciążek-Jurczyk, M.; Sulkowska, A. Spectroscopic analysis of the impact of oxidative stress on the structure of human serum albumin (HSA) in terms of its binding properties. Spectrochimica Acta Part A: Molecular and Biomolecular Spectroscopy 2015, 136, 265-282.

4. Carter, D.C.; Ho, J.X. Structure of serum albumin. In Advances in protein chemistry; Elsevier, 1994; Vol. 45, pp. 153-203.

5. He, X.M.; Carter, D.C. Atomic structure and chemistry of human serum albumin. Nature 1992, 358, 209.

6. Waheed, A.A.; Rao, K.S.; Gupta, P.D. Mechanism of Dye Binding in the Protein Assay Using Eosin Dyes. Analytical Biochemistry 2000, 287, 73-79, doi:10.1006/abio.2000.4793.

7. Gooijer, C. Introduction to Fluorescence Spectroscopy. Analytica Chimica Acta 2007, 419, 116-117.

8. Katrahalli, U.; Jaldappagari, S.; Kalanur, S.S. Study 
of the interaction between fluoxetine hydrochloride and bovine serum albumin in the imitated physiological conditions by multi-spectroscopic methods. Journal of Luminescence 2010, 130, 211216, doi:10.1016/j.jlumin.2009.07.033.

9. Chaturvedi, S.K.; Ahmad, E.; Khan, J.M.; Alam, P.; Ishtikhar, M.; Khan, R.H. Elucidating the interaction of limonene with bovine serum albumin: a multitechnique approach. Mol. BioSyst. 2015, 11, 307-316, doi:10.1039/C4MB00548A.

10. Beauchemin, R.; N'Soukpoe-Kossi, C.N.; Thomas, T.J.; Thomas, T.; Carpentier, R.; Tajmir-Riahi, H.A. Polyamine analogues bind human serum albumin. Biomacromolecules 2007, 8, 3177-3183.

11. Ross, P.D.; Subramanian, S. Thermodynamics of protein association reactions: forces contributing to stability. Biochemistry 1981, 20, 3096-3102, doi:10.1021/bi00514a017.

12. Bordbar, A.K.; Taherikafrani, A. Binding and fluorescence study on interaction of human serum albumin (HSA) with cetylpyridinium chloride (CPC). Colloids \& Surfaces B Biointerfaces 2007, 55, 84-89.

13. Reynolds, D.M. Rapid and direct determination of tryptophan in water using synchronous fluorescence spectroscopy. Water Research 2003, 37, 3055-3060, doi:10.1016/S0043-1354(03)00153-2.

14. Watanabe, H.; Tanase, S.; Nakajou, K.; Maruyama, T.; Kragh-Hansen, U.; Otagiri, M. Role of Arg-410 and Tyr-411 in human serum albumin for ligand binding and esterase-like activity. Biochemical Journal 2000, 349, 813-819, doi:10.1042/bj3490813.

15. Wang, Y.Q.; Tang, B.P.; Zhang, H.M.; Zhou, Q.H.; Zhang, G.C. Studies on the interaction between imidacloprid and human serum albumin: spectroscopic approach. Journal of Photochemistry \& Photobiology B Biology 2009, 94, 183-190. 